\section{Commentary}

A reliable, efficient, and simple method for spinal cord protection is sorely needed. The article by Follis and associates reports a novel approach toward developing such a method. Considering the popularity that retrograde perfusion of the cerebral venous circulation has achieved in brain protection, it is not surprising that a similar approach is now tried for spinal cord protection. The design of the experimental protocol, however, suggests that it is based on 2 important misconceptions about the effect of retrograde brain perfusion: (1) that retrograde perfusion in fact does reach the brain tissue in quantity to supply nutritive flow of useful proportions to support metabolism under normothermic conditions and (2) that the observed protective effect of retrograde cerebral perfusion in the brain is based primarily on the supply of nutritive blood flow rather than the efficient local cooling of the brain by the accompanied deep hypothermia.

The evidence in the literature proves that both of these premises are false. Application of these concepts to the protection of the spinal cord is further compounded by the widely known characteristics of the cord arterial blood supply. In the presence of rich venous and arterial connections of the cord circulation, it is only logical to expect the retrograde blood flow from the azygos system to drain away from the highresistance arterial bed of the cord tissue into the empty low-resistance descending aorta and ischemic lower body. Follis and colleagues, in their study, have elegantly demonstrated that the foregoing considerations are sound and normothermic retrograde perfusion of the azygos system does not protect the spinal cord during periods of diminished flow. Providing hypothermic flow into the azygos system is an intriguing idea and may be an efficient way of cooling the spinal cord. However, the benefits of local venous cooling may well be offset by intraspinal venous hypertension, the accompanied cord edema, and impaired postischemic reperfusion. Whether hypothermic retrograde venous perfusion of the spinal cord is a practical method superior to those already in use (epidural cooling, deep hypothermic circulatory arrest, regional arterial cooling, and permissive hypothermia) remains to be seen.

M. Arisan Ergin, $M D$ New York, New York

12/1/101558

\title{
Availability of Journal back issues
}

As a service to our subscribers, copies of back issues of The Journal of Thoracic and Cardiovascular Surgery for the preceding 5 years are maintained and are available for purchase from Mosby at a cost of $\$ 17.00$ per issue until inventory is depleted. The following quantity discounts are available: $25 \%$ off on quantities of 12 to 23, and one third off on quantities of 24 or more. Please write to Mosby, Inc, Subscription Services, 11830 Westline Industrial Drive, St Louis, MO 63146-3318, or call 800-453-4351 or 314-453-4351 for information on availability of particular issues. If unavailable from the publisher, photocopies of complete issues may be purchased from Bell \& Howell Information and Learning, 300 N Zeeb Rd, Ann Arbor, MI 48106-1346, 734-761-4700. 